\title{
An Algorithm for the Generalized $k$-Keyword Proximity Problem and Finding Longest Repetitive Substring in a Set of Strings
}

\author{
Inbok Lee ${ }^{1, \star}$ and Sung-Ryul Kim ${ }^{2, \star \star}$ \\ 1 King's College London, Department of Computer Science, \\ London WC2R 2LS, United Kingdom \\ inboklee@gmail.com \\ 2 Division of Internet \& Media and CAESIT, \\ Konkuk University, Seoul, Republic of Korea \\ kimsr@konkuk.ac.kr
}

\begin{abstract}
The data grid may consist of huge number of documents and the number of documents which contain the keywords in the query may be very large. Therefore, we need some method to measure the relevance of the documents to the query. In this paper we propose algorithms for computing $k$-keyword proximity score $[3]$ in more realistic environments. Furthermore, we show that they can be used to find longest repetitive substring with constraints in a set of strings.
\end{abstract}

\section{Introduction}

The data grid may consist of huge number of documents and the number of documents which contain the keywords in the query may be very large. All these documents may not be relevant to what the user wants: some may contain them in different contexts. Therefore, we need a method to measure the relevance of the documents to the query. Here we focus on the proximity of the keywords which means how close they appear together in the document. If they are appearing close (good proximity), it is likely that they have stronger combined meaning.

The offset of a word in a document is the distance (number of the words) from the start of the document. A range $[a, b]$ in a document represents the contiguous part of the document from $a$-th word to $b$-th word in the document. The size of the range $[a, b]$ is $b-a$.

In the data grid, we assume that documents are stored in inverted file structure. Each keyword has a list of IDs of documents which contain the keyword and a sorted list of offsets in the document. Using inverted file structure, we can easily obtain the set of documents which contain the keywords.

Kim et al. [3] defined the generalized proximity score and proposed $O(n \log k)$ time algorithm where $k$ is the number of keywords and $n$ is the number of occurrences of the keywords in the document.

\footnotetext{
^ This work was supported by the Post-doctoral Fellowship Program of Korea Science and Engineering Foundation (KOSEF).

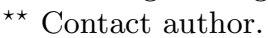


Definition 1. Given $k$ keywords $w_{1}, w_{2}, \ldots, w_{k}$, a set of lists $K=\left\{K_{1}, K_{2}, \ldots K_{k}\right.$ where each keyword $w_{i}$ has a sorted list of offsets $K_{i}=\left\{o_{i 1}, o_{i 2}, \ldots\right.$, oij $_{i}(1 \leq$ $i \leq k)$, and $k$ positive integers $R_{1}, R_{2}, \ldots, R_{k}$, and another integer $k^{\prime}(\leq k)$, the generalized $k$-keyword proximity problem is finding the smallest range that contains $k^{\prime}$ distinct keywords where each $w_{i}$ of these keywords appears at least $R_{i}$ times.

We briefly explain Kim et al.'s algorithm. A more detailed account can be found in [3]. We need to define two terms.

Definition 2. A candidate range is a range which contains at least $k^{\prime}$ distinct keywords where each keyword $w_{i}$ appears at least $R_{i}$ times in the range.

Definition 3. A critical range is a candidate range which does not contain another candidate range.

We can easily show that the solution is the smallest critical range. Hence we need to find critical ranges and report the smallest one. First, we merge the sorted lists of offsets of $k$ keywords into one list. This step runs in $O(n \log k)$ time. For simplicity we assume that each offsets are mapped to a number in range $[1 . . n]$. We store the keyword ID into a list $L[1 . . n]$.

The outline of the algorithm is that we first find a candidate range (expanding sub-step) and find a critical range from that candidate range (shrinking sub-step).

We maintain a range $[a, b]$. Initially $a=1$ and $b=0$. We also maintain $k$ counters $c_{1}, c_{2}, \ldots, c_{k}$. Initially $c_{1}=c_{2}=\cdots=c_{k}=0$. And we maintain a counter $h$ which counts the number of $c_{i}$ 's $(1 \leq i \leq k)$ that are $\geq R_{i}$. Initially $h=0$.

In the expanding sub-step, the range is expanded from $[a, b]$ to $[a, b+1]$. We check $L[b]$ and set $c_{L[b]}=c_{[L[b]}+1$. We also check whether $c_{L[b]}=R_{i}$. If so, we update $h=h+1$. We repeat this loop until $h=k^{\prime}$. Then $[a, b]$ is a candidate range and go to the shrinking sub-step.

In the shrinking sub-step, the range is reduced from $[a, b]$ to $[a+1, b]$. We also set $c_{L[a]}=c_{L[a]}-1$ and check whether $c_{L[a]} \leq R_{L[a]}$. If so, $h=h-1$. And if $h<k^{\prime}$, we report a critical range $[a-1, b]$. We go back to the expanding sub-step with the range $[a, b]$. These steps run in $O(n)$ time and the total time complexity is $O(n \log k)$.

\section{Our Improvements}

Here we can consider the following variations. First, the original problem does not specify the keyword which should be included. Some keywords may be more important than others.

Problem 1. In $k$-keyword proximity problem, one special keyword $w_{i}$ in the query should appear in the range.

Without loss of generality, assume the keyword that must appear in the critical range is $w_{1}$. The original algorithm may report no critical range with $w_{1}$ even though the document contains $w_{1}$ ! 
We first find a candidate for the problem and make the range narrow as much as possible. In the expanding sub-step, we move to the shrinking sub-step only after the current range $[a, b]$ contains $w_{1}$ at least $R_{1}$ times. We guarantee that the input to the shrinking sub-step meets the constraints of Problem 1 . In the shrinking sub-step, we add one more check. If, by shrinking from $[a, b]$ to $[a+1, b]$, $c_{1}$ becomes smaller than $R_{1}$, we report $[a, b]$ as a critical range (without checking the condition $h<k^{\prime}$ ).

Now we consider another problem when keywords in the query must be in some order (for example, "Paris Hilton" and "Hilton Paris").

Problem 2. A keyword $w_{i}$ must appear before another keyword $w_{j}$.

Without loss of generality, assume that $w_{2}$ must follow $w_{1}$. It means that $w_{2}$ can appear only after $w_{1}$ appears at least $R_{1}$ times. In the expanding sub-step, we move to the shrinking sub-step only after the current range $[a, b]$ contains $w_{1}$ at least $R_{1}$ times before $w_{2}$ appears. We may encounter $w_{2}$ before $R_{1} w_{1}$ 's. Then we discard the current range $[a, b]$. We restart the expanding sub-step with the range $[b+1, b+1]$ and initialize all the variables. In the shrinking sub-step, we do the same as we did in Problem 1.

Finally, we can consider the case where two keywords have a Boolean relation.

Problem 3. If a keyword $w_{i}$ appears in the document, then also another keyword $w_{j}$ must/must not appear in the document (AND/XOR relation).

Without loss of generality, assume that $w_{1}$ and $w_{2}$ forms these relations. First we consider the AND relation. In the expanding sub-step, we maintain a flag $f$. Initially $f=0$. When we meet a $w_{1}$, we set $f=1$. When we move to the shrinking sub-step in the original algorithm, we check whether the flag $f$ is ON. If so, we postpone until we meet an occurrence of $w_{2}$. In the shrinking sub-step, we use the flag again. If $f=0$, there is no modification at all. But if $f=1$, each time we shrink the range we check whether it removes the occurrence of $w_{1}$. If so, we report the range $[a, b]$ as the critical range.

The procedure is almost the same when we handle the XOR relation. We use the flag again. When we meet an occurrence of $w_{1}$, we set $f=1$. If we meet an occurrence of $w_{2}$ and $f=1$, then we discard the current range $[a, b]$ and restart with $[b+1, b+1]$. The shrinking sub-step is the same as the original algorithm.

All these modification does not change the time complexity.

Theorem 1. All the problems in Section 2 can be solved in $O(n \log k)$ time.

\section{Repetitive Longest Substring in a Set of Strings}

Now we consider finding the longest substring in a set of strings.

Problem 4. Given a set of strings $\mathcal{U}=\left\{T_{1}, T_{2}, \ldots, T_{k}\right\}$, a set of positive integers $\mathcal{D}=\left\{d_{1}, d_{2}, \ldots, d_{k}\right\}$, and a positive integer $k^{\prime}(\leq k)$, find the longest sting $w$ which satisfies two conditions: (a) there is a subset $\mathcal{U}^{\prime}$ of $\mathcal{U}$ such that $w$ appears at least $d_{i}$ times in each string $T_{i}$ in $\mathcal{U}^{\prime}$, and (b) $\left|\mathcal{U}^{\prime}\right|=k^{\prime}$. 
We use the suffix array. The suffix array of a text $T$ is a sorted array suf $[1 . .|T|]$ and $l c p[1 . .|T|]$. suf $[k]=i$ if and only if $T[i . .|T|]$ is the $k$-th suffix of $T$. $l c p[k]$ is the length of the longest common prefix between each substring in the suffix array and its predecessor and $l c p(a, b)=\min _{a \leq i \leq b} l c p[i]$ with the following properties.

Fact 1. $l c p(a, b) \leq l c p\left(a^{\prime}, b^{\prime}\right)$ if $a \leq a^{\prime}$ and $b \geq b^{\prime}$.

Fact 2. The length of the longest common prefix of $T[$ suf $[a] . .|T|], T[$ suf $[a+$ $1] . .|T|], \ldots, T[$ suf $[b] . .|T|]$ is $\operatorname{lcp}(a+1, b)$.

To build the suffix array for $\mathcal{U}=\left\{T_{1}, T_{2}, \ldots, T_{k}\right\}$, we create a new string $T^{\prime}=T_{1} \% T_{2} \% \cdots T_{k}$ where $\%$ is a special symbol and is smaller than any other character in $\Sigma$. sufand lcparrays can be computed in $O\left(\left|T^{\prime}\right|\right)$ time by [2,1] with one modification: \% does not match itself. We use another array $i d s$. $i d s[j]=i$ if $T^{\prime}[j] T^{\prime}[j+1] \cdots \%$ was originally a suffix of $T_{i}$ (we mean the first \% after $T^{\prime}[j]$ ). This computation also takes $O\left(\left|T^{\prime}\right|\right)$ time.

We briefly explain the outline of [4. Fact 1 tells that the smaller a range becomes, the longer the common prefix is. Hence, we consider IDs of strings as IDs of keywords as we did in Section 2. What we need is the smallest range that yields the longest common prefix of the suffixes (the longest common substring). We use the same algorithm in Section 2, without the merging step. Hence the time complexity is $O(n)$.

The problems in Section 2 can be transformed into these following problems except Problem 2 because we do not consider order in $\mathcal{U}$.

Problem 5. The same as Problem 4, but $\mathcal{U}^{\prime}$ must contain $T_{i}$.

Problem 6. The same as Problem 4 , but if $\mathcal{U}^{\prime}$ contains a string $T_{i}$, it must/must not contain another string $T_{j}$ (AND/XOR relation).

All these problems can be solved in $O(n)$ time with equivalent algorithm in Section 2.

\section{References}

1. T. Kasai, G. Lee, H. Arimura, S. Arikawa, and K. Park. Linear-time longestcommon-prefix computation in suffix arrays and its applications. In CPM 2001, pages 181-192, 2001.

2. D. K. Kim, J. S. Sim, H. Park, and K. Park. Linear-time construction of suffix arrays. In CPM 2003, pages 186-199, 2003.

3. S.-R. Kim, I. Lee, and K. Park. A fast algorithm for the generalised $k$-keyword proximity problem given keyword offsets. Information Processing Letters, 91(3):115120,2004 .

4. I. Lee, and Y. J. Pinzon Ardilà. Linear time algorithm for the generalised longest common repeat problem. In SPIRE 2005, pages 191-200, 2005. 